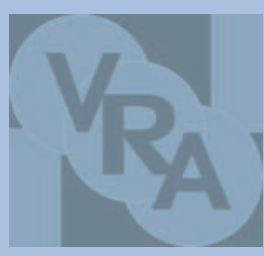

\title{
Neue Gesetze und Sonderregelungen zur Unterstützung des Gesundheitswesens bei der Bewältigung der Corona-Epidemie in Deutschland
}

Nach Beschluss des Bundestages am 25.03.2020 und Zustimmung durch den Bundesrat am 27.03.2020 stehen 2 Gesetzespakete zur Stärkung der Gesundheitsversorgung der Bevölkerung in Deutschland zur Verfügung.

Mit dem „Covid-19-Krankenhausentlastungsgesetz" sollen die wirtschaftlichen Folgen für Krankenhäuser und Vertragsärzte abgefangen werden.

Mit dem „Gesetz zum Schutz der Bevölkerung bei einer epidemischen Lage von nationaler Tragweite" soll die Reaktionsfähigkeit des Ministeriums für Gesundheit auf Epidemien verbessert werden.

Ziel des gesundheitspolitischen Handelns ist die Kompensation von Einnahmeausfällen, der Bürokratieabbau, das Aussetzen von Sanktionen sowie die Beschleunigung von Entscheidungsprozessen in epidemischen Lagen. Parallel zum skizzierten gesundheitspolitischen Handeln mit neuen Gesetzespaketen hat der Gemeinsame Bundesausschuss (G-BA) im Zusammenhang mit der Pandemie mit SARS-CoV-2 zusätzlich zeitlich befristete Sonderregelungen getroffen mit dem Ziel, Krankenhäuser und Praxen zu entlasten und Infektionsrisiken für Patienten zu verringern.

\section{Eckpunkte des „Covid-19- Krankenhausentlastungs- gesetzes":}

Mit der neuen Gesetzgebung sollen die Versorgungskapazitäten für eine wachsende Anzahl von Menschen mit einer Corona-Virus-Infektion nach oben angepasst werden.

Finanzielle Einbußen der Krankenhäuser, der Vertragsärzte wie auch der Pflegeeinrichtungen sollen abgefedert werden.
Beispielhaft nachstehend einige Maßnahmen (s. Bundesgesetzblatt Jahrgang 2020, Teil I, Nr. 14, ausgegeben zu Bonn am 27. März 2020):

- Rückwirkend ab dem 16.03.2020 bis zum 30.09.2020 erhalten Krankenhäuser für jedes im Verhältnis zum Vorjahr „freie“ Bett (voll- oder teilstationär) eine tagesbezogene Pauschale in Höhe von $560,00 €$.

- Zugelassene Krankenhäuser erhalten bei Ausweitung ihrer intensivmedizinischen Behandlungskapazitäten für jedes neue intensivmedizinische Bett mit maschineller Beatmungsmöglichkeit jeweils $50000 €$ bis zum 30.09.2020.

- Für jeden voll- oder teilstationär versorgten Patienten im Zeitraum vom 01.04 .2020 bis 30.06.2020 erhalten die Krankenhäuser einen Zuschlag in Höhe von 50,00€ für mit Schutzkleidung verbundene Preis- und Mengensteigerungen.

Die vorbeschriebenen Anpassungen - ausgelöst durch die Corona-19-Epidemie in Deutschland - wurden als Änderungen des Krankenhausfinanzierungsgesetzes im Artikel 1 des Covid-19-Krankenhausentlastungsgesetzes aufgelistet.

Die nachstehend beispielhaft aufgeführten Maßnahmen werden beschrieben unter $\mathrm{Ar}$ tikel 2 des Gesetzes mit dem Titel: „Änderungen des Krankenhausentgeltgesetzes“:

- Der Fixkostendegressionsabschlag gilt nicht für die Vereinbarung des Erlösbudgets für das Jahr 2020.

- Der sogenannte „vorläufige Pflegeentgeltwert" wird von 146,55€ auf $185,00 €$ pro Pflegetag erhöht.

- Im Artikel 3 des Covid-19-Krankenhausentlastungsgesetzes finden sich unter dem Titel: „Änderungen des 5. Buches im Sozialgesetzbuch“ weitere Beispiele entsprechender gesetzlicher Regelungen mit Fokus auf die Covid-19-Epidemie in Deutschland.

- Niedergelassene Ärzte sowie Psychotherapeuten werden bei einer zu hohen Umsatzminderung (> 10\% im Vergleich zum Vorjahr) aufgrund einer geringeren Inanspruchnahme durch Patienten mit Ausgleichszahlungen sowie mit zeitnahen Anpassungen der Honorarverteilung geschützt.

- Vorsorge- und Rehabilitationseinrichtungen erhalten für einen befristeten Zeitraum einen anteiligen finanziellen Ausgleich für nicht belegte Betten.

- Die von den Krankenhäusern bis zum 31.12.2020 erbrachten und in Rechnung gestellten Leistungen sind von den Krankenkassen innerhalb von 5 Tagen nach Rechnungseingang zu bezahlen.

- Pflegeeinrichtungen wird durch eine entsprechende Regelung die Sicherheit gegeben, die durch die Epidemie bedingten, außerordentlichen Aufwendungen oder Einnahmeausfälle über die Pflegeversicherung erstattet zu bekommen.

Eckdaten des „Gesetzes zum Schutz der Bevölkerung bei einer epidemischen Lage von zentraler Tragweite“

(s. Bundesgesetzblatt Jahrgang 2020, Teil I, Nr. 15, herausgegeben zu Bonn am 27.03.2020).

Mit diesem Gesetz wird das Bundesministerium für Gesundheit u. a. ermächtigt, durch Allgemeinverfügungen oder Rechtsverordnungen Vorkehrungen zum Schutz der Bevölkerung zu treffen und die Gesundheitsversorgung sicherzustellen, z. B. durch: 
- Vorschriften für den grenzüberschreitenden Reiseverkehr

- Melde- und Untersuchungspflichten

- Maßnahmen zur Sicherstellung der Grundversorgung mit Arzneimitteln, Schutzausrüstung und Labordiagnostik

\section{G-BA beschließt weit- reichende Maßnahmen zur Entlastung von Kranken- häusern und Ärzten so- wie zum Schutz vor Infektionsrisiken}

Im Zusammenhang mit der Epidemie mit SARS-CoV-2 erklärte Herr Prof. Hecken (Unparteiischer Vorsitzender des G-BA) am 27.03.2020:

„In der jetzigen Ausnahmesituation müssen alle verfügbaren personellen Ressourcen beim Pflegepersonal und bei den Ärztinnen und Ärzten für die Patientenversorung frei gemacht werden und deshalb Bürokratie und Dokumentationsaufgaben zur Qualitätssicherung auf ein unabdingbares Minimum reduziert werden...".

„...Mit den beschlossenen Sonderregelungen sollen die notwendige Flexibilität und Handlungsfreiheit im Ressourceneinsatz den Vertragsärzten und Krankenhäusern eingeräumt werden. Ziel ist es, die Infektionsrisiken für Patienten sowie für das ärztliche und pflegerische Personal zu minimieren..."

Die getroffenen und befristet geltenden Sonderregelungen fokussieren sich beispielhaft auf nachstehende Richtlinien bzw. Regelungen.

\section{Flexiblere Verordnungs- möglichkeiten durch Krankenhäuser}

Im Rahmen des Entlassmanagements können nicht nur bis zu 7, sondern nunmehr bis zu 14 Tage nach Entlassung aus dem Krankenhaus häusliche Krankenpflege, spezialisierte ambulante Palliativversorgung, Sozio-Therapie sowie Heil- und Hilfsmittel verordnet werden.

\section{Regelungen zu einem}

\section{gestuften System von Notfall-} situationen in Krankenhäusern

Ziel der neuen Regelung ist die „schnellstmögliche Aufnahmebereitschaft für beatmungspflichtige Intensivpatienten" (nicht mehr 60 Minuten nach Krankenhausaufnahme).

\section{Weitere Ausnahmen von}

\section{Anforderungen an die Quali- tätssicherung}

Ausnahmen von Anforderungen an die Qualitätssicherung wurden beschlossen. Diese umfassen u. a.:

- Änderungen der Regelung zur Datenvalidierung,

- zum strukturierten Dialog und

- zum Stellungnahmeverfahren.

\section{Zentrumsregelungen}

Krankenhäuser, die bereits vor Inkrafttreten der Zentrumsregelungen im Krankenhausplan besondere Aufgaben wahrgenommen haben, haben weitere 6 Monate Zeit, die vorgegebenen Qualitätsanforderungen umzusetzen.

Bereits am 20.03.2020 hatte der G-BA Abweichungsmöglichkeiten von der Mindestausstattung mit Intensivpflegepersonal bei bestimmten komplexen Behandlungen beschlossen.

\section{Resümée}

Mit Schreiben vom 13.03.2020 forderte Bundesgesundheitsminister Jens Spahn die Geschäftsführungen der Krankenhäuser auf, alle planbaren Aufnahmen, Opera- tionen und Eingriffe ab Montag, 16.03.2020 auf unbestimmte Zeit zu verschieben, wodurch in der überwiegenden Zahl deutscher Krankenhäuser entsprechend freie Ressourcen für die Aufnahme von Covid-19-Patienten vorgehalten werden können.

Inwieweit das Covid-19-Krankenhausentlastungsgesetz die zu erwartenden Verluste der Krankenhäuser, der Vertragsärzte sowie der Rehakliniken und Pflegeeinrichtungen mit den dort beschriebenen Maßnahmen ausgleichen kann, muss für viele Einrichtungen mehr als kritisch gestellt werden.

Es ist zu hoffen, dass nach der Covid-19-Krise entsprechend sachgerecht und angemessen entschieden wird und sich hieraus ableitet, dass ein „weiter so“ “ wie vor der Krise der falsche Weg für ein zukünftig leistungsfähiges Gesundheitswesen in Deutschland ist.

Prof. Dr. med. Heinz-Jürgen Lakomek Geschäftsführer VRA

Dr. med. Joachim Abrolat

1. Stellvertretender Vorsitzender VRA

Dr. med. Ansgar Klemann Schatzmeister VRA

\section{KONTAKTADRESSE}

Verband Rheumatologischer Akutkliniken e. V.

Geschäftsstelle

Rechtsanwaltskanzlei Meyer-Koering

Schumannstr. 18, 10177 Berlin

Tel.: 030/2062 98-79/-82

E-Mail: gf@vraev.de

Internet: www.vraev.de

\section{IMPRESSUM}

\section{Verantwortlich für den Inhalt}

Prof. Dr. Heinz-Jürgen Lakomek, Direktor, Universitätsklinik für Geriatrie, Johannes Wesling Klinikum Minden 